$>$ En 1982, S.B. Prusiner a émis l'hypothèse selon laquelle l'agent infectieux des encéphalopathies spongiformes était uniquement constitué d'une protéine, la protéine prion. Celle-ci, une protéine cellulaire normale, peut adopter une conformation pathologique qui, ensuite, convertit la forme normale en forme pathologique, la protéine infectieuse. Cette hypothèse pose de nombreux problèmes relevant de la biologie structurale des protéines. Nos connaissances sur la structure spatiale et le repliement de la protéine prion ont progressé de façon importante et nous essayons de concilier les résultats expérimentaux actuels avec l'hypothèse prion. Si la structure de la protéine recombinante ne révèle rien de particulier, son repliement dans des conditions acides permet d'atteindre une structure en feuillet $ß$ similaire à celle de la protéine trouvée chez les animaux infectés, mais qui, malheureusement, n'est pas infectieuse. La participation de co-facteurs comme les chaperons est évoqué, mais n'a pas permis d'atteindre la structure infectieuse. L'analyse des résultats expérimentaux ne permet ni d'infirmer ni de confirmer l'hypothèse prion, mais des aspects importants restent encore à explorer.

\section{La protéine prion : structure, dynamique et conversion in vitro}

Jean-Pierre Liautard, Maria-Teresa Alvarez-

Martinez, Cécile Féraudet, Joan Torrent

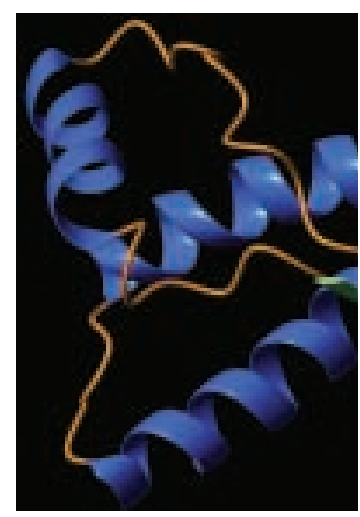

PrPsc. Sous l'angle de la biologie structurale, les deux concepts majeurs de cette hypothèse sont : 1) l'existence de deux conformations pour une même séquence de protéine; et 2) la possibilité qu'aurait la protéine pathologique de convertir la forme normale en forme pathologique, le "baiser de la mort" comme l'a qualifié B. Caughey [3]. Quels sont aujourd'hui les arguments en faveur de ces hypothèses, ou plutôt dans quelle mesure les résultats expérimentaux s'accordent-ils avec l'hypothèse prion? Pour cela, nous examinerons la (ou les) structure(s) de la protéine prion, son repliement in vitro et in vivo et les systèmes de conversion acellulaires.

\section{Structures spatiales de la protéine prion} fiques; en témoigne l'attribution en 1997 du prix Nobel à son auteur. Mais de l'admission comme hypothèse de travail à la démonstration expérimentale définitive, il existe un fossé qui n'a pas encore été entièrement comblé. Examinons l'aspect structural de la proposition de S.B. Prusiner dans sa version actuelle car, depuis 1982, elle a fait l'objet de nombreuses adaptations [1,2]. Une protéine cellulaire, la protéine prion ( $\mathrm{PrPC})$, existe sous deux conformations (deux types de repliements). La première est la forme cellulaire naturelle (normale ou PrPC), la seconde est infectieuse car elle induit à son avantage la conversion de la forme normale, et participe à la pathogénie. Cette forme est souvent nommée
La protéine prion humaine, codée par un seul exon, est formée initialement de 254 acides aminés (Figure 1). Elle subit ensuite une maturation, c'est-à-dire l'élimination d'une séquence signal de 22 résidus, localisée à son extrémité $\mathrm{N}$-terminale et d'une autre séquence de 23 acides aminés à son extrémité $\mathrm{C}$-terminale, permettant alors l'ajout d'une ancre glycosylphosphatidylinositol (GPI). Ainsi, la PrPC est constituée de 209 acides aminés. Un pont disulfure est formé entre les cystéines 179 et 214. La protéine est glycosylée sur les asparagines 181 et 197 mais la glycosylation n'est pas néces- 
saire à l'infectiosité (voir glossaire). La protéine normale est donc exposée à l'extérieur de la cellule, retenue dans la membrane par son ancre GPI. Dans la partie $\mathrm{N}$-terminale de la molécule, on remarque une répétition imparfaite (5 fois) d'un octapeptide. Cette région contient des histidines qui chélatent les ions cuivres et jouent un rôle important dans des phénomènes d'oxydoréduction. Ces caractéristiques structurales ne sont pas l'apanage de la protéine humaine: les protéines prion des différentes espèces de mammifères sont très semblables et présentent une forte conservation de leur séquence et une organisation générale identique.

Répondre à la question de la structure spatiale de la protéine prion n'est pas simple car la protéine est susceptible d'exister sous plusieurs conformations. De plus, les méthodes d'obtention de la conformation sont cruciales. Aujourd'hui, la seule forme dont la structure a été déterminée au niveau atomique est la protéine recombinante $(\mathrm{rPrP})$ produite dans $\varepsilon$. coli et repliée in vitro. Une représentation schématique de l'arrangement dans l'espace déterminé par RMN (résonance magnétique nucléaire) [4] est montrée dans la figure 2. Deux domaines sont immédiatement décelables : 1) un cœur structuré (aa 120 à 230) présentant 3 hélices $\alpha$ et un petit feuillet $\beta$ formé de deux chaînes antiparallèles; 2) une longue chaîne flexible à l'extrémité $\mathrm{N}$-terminale qui ne présente pas de structure définie en solution. 0 n peut souligner que les mutations qui sont à l'origine des formes génétiques de la maladie humaine (voir glossaire) sont principalement localisées dans les hélices $\alpha$

(Figure 1). II n'existe cependant pas de corrélation entre ces mutations et la stabilité de la structure correspondante [5].

De fait, on ne connaît de façon sûre et définitive ni la structure de la forme normale (PrPc) ni celle de la forme pathologique ( $P r P s c)$. Celle de la $\operatorname{PrPC}$ est inconnue car on n'a pas encore réussi à en purifier des quantités suffisantes pour déterminer sa structure par RMN ou pour parvenir à la cristalliser. En revanche, des informations indirectes sur le nombre d'hélices $\alpha$ et de feuillets $ß$ ont été obtenues : les quantités respectives de ces deux structures secondaires sont proches de celles de la protéine recombinante (Tableau I). On admet donc, mais sans preuve définitive, que la protéine normale ( $P r P C$ ) présente une structure proche, sinon identique, à celle de la protéine recombinante. La protéine pathologique ( $\mathrm{PrPs}$ ), qui s'agrège sous forme d'amyloïdes (voir glossaire), est en revanche très riche en feuillets $\beta$ (Tableau 1). L'agrégation de cette forme rend les études par RMN ou par diffraction des rayons $X$ pratiquement impossibles et obère une possible détermination de la structure au niveau atomique. Quoi qu'il en soit, elle apparaît très différente de la forme PrPC. Une propriété particulière et étonnante semble liée à la structure (fine) de la et insomnie fatale familiale (IFF). En outre, plusieurs polymorphismes de la protéine sont connus. 
forme pathologique, il s'agit de l'existence de souches (ou isolats, voir glossaire). Les souches de l'agent infectieux prions sont corrélées, au moins partiellement, à des propriétés biochimiques (résistance à la protéinase $K$, mobilité électrophorétique, stabilité) qui bien évidemment reflètent des structures spatiales différentes. La conjecture prion est donc assortie de l'hypothèse selon laquelle les caractéristiques biologiques et pathologiques des souches sont cryptées dans la structure spatiale de la PrPSc. En conséquence, ces différences subtiles de conformation doivent être reproduites lors de la conversion de la forme PrPC en PrPSc.

Ainsi, la détermination des structures spatiales des deux formes naturelles de la protéine prion, c'est-à-dire PrPC et PrPsc, reste encore aujourd'hui un des objectifs majeurs de recherche.

\section{Analyse du repliement de la protéine prion in vitro}

Une autre possibilité d'obtenir des indications sur l'existence de deux structures différentes de la protéine prion est d'étudier in vitro le repliement de la protéine. Par exemple, peut-on mettre en évidence des intermédiaires de repliement, voire obtenir les différentes conformations dans les conditions "normales" ou en modifiant les conditions physico-chimiques et thermodynamiques lors du repliement? De nombreux travaux ont été entrepris pour tenter de résoudre cette question, et tous utilisent la protéine recombinante $r \operatorname{PrP}$ produite dans $\varepsilon$. coli. La dénaturation de la protéine par le chlorure de guanidinium (CIGdn) est parfaitement réversible, et a permis de calculer l'énergie de stabilisation de la molécule qui est de $22 \mathrm{KJ} / \mathrm{mol}$. Cela représente une valeur faible mais pas rare. La dénaturation par la chaleur est irréversible car, dans ces conditions, la protéine dénaturée forme des agrégats. Ce phénomène n'est pas exceptionnel et il est donc difficile d'en tirer des conclusions spécifiques. Lorsque I'on renature la protéine par dilution de l'agent dénaturant ( $\mathrm{ClGdn}$ ), on observe un repliement extrêmement rapide qui ne laisse apparaître aucun intermédiaire à partir duquel une nouvelle structure pourrait être atteinte [6]. En effet, à $4^{\circ} \mathrm{C}$, la demi-vie pour le repliement est de $170 \mu$ s, ce qui représente certainement la vitesse la plus rapide enregistrée à ce jour. On peut donc conclure que, dans un environnement mimant les conditions physiologiques $(\mathrm{pH}=7)$, il n'existe aucun argument en faveur de la présence d'un intermédiaire de repliement. La protéine prion ne semble donc pas présenter des caractéristiques qui en feraient une protéine originale capable de présenter deux structures différentes.

$\varepsilon$ n revanche, des propriétés singulières de la protéine

\begin{tabular}{lccc}
\hline Protéine & $\%$ hélice $\alpha$ & \% hélice B & \% aléatoire \\
PrPc & 42 & $3-5$ & $53-55$ \\
PrPSc & 30 & 43 & 27 \\
PrPSc 27-30 & 25 & 54 & 25 \\
rPrP & 28,5 & 3 & 68 \\
\hline
\end{tabular}

Tableau 1. Pourcentage d'hélice $\alpha$ et de feuillet $B$ dans les différentes formes de la protéine prion. Ce tableau représente la compilation des résultats obtenus par différents laboratoires utilisant des méthodes qui peuvent être différentes. Sont principalement utilisés le dichroïsme circulaire pour les formes solubles et l'infrarouge pour les formes solubles ou non. Pour la protéine recombinante, les structures ont été aussi calculées à partir de la structure RMN. La quantité mesurée doit être considérée comme approximative principalement en raison des contaminations. Par exemple, la forme PrPsc est un agrégat amyloïde qui emprisonne de nombreuses molécules extrêmement difficiles à éliminer. $\mathrm{Pr} P \mathrm{C}$ correspond à la protéine normale extraite de cerveau alors que rPrP est la protéine recombinante. PrPSc est la protéine pathologique extraite de cerveau sans utilisation de protéinase K. PrPsc27-30 est la protéine pathologique extraite de cerveau après traitement à la protéinase $\mathrm{K}$ qui a enlevé la partie $\mathrm{N}$-terminale jusqu'à environ le résidu 90 .

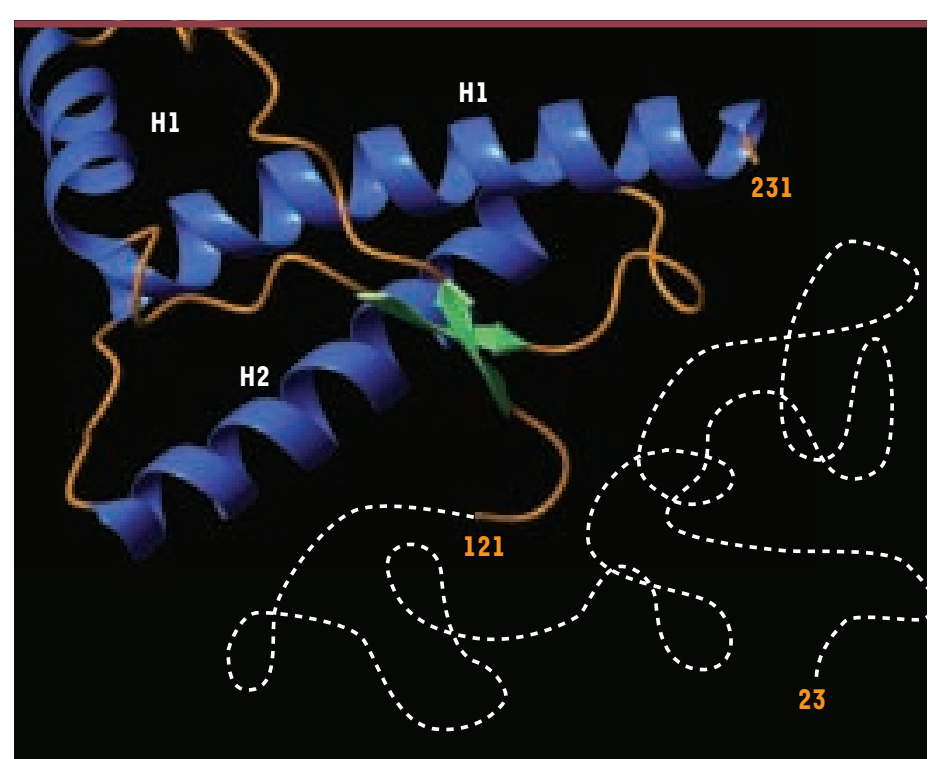

Figure 2. Représentation schématique de la structure tridimensionnelle de la protéine prion normale recombinante de hamster. La molécule est formée d'une chaîne polypeptidique repliée de 110 acides aminés (résidus 121 à 231) qui présente trois hélices $\alpha$ (en bleu) et deux petits feuillets $\beta$ anti-parallèles (en vert). Les boucles de connexion sont représentées en orange. Alors que l'extrémité C-terminale de la protéine est structurée, la partie $\mathrm{N}$-terminale (résidus 23 à 121) est mobile (ligne blanche pointillée). Aucune structure définie n'est observée en RMN. 
ont été mises en évidence dans des conditions très particulières. Il existe en effet, dans la protéine normale, un pont disulfure entre les hélices 2 et 3 (Figure I). Généralement, les ponts disulfures ne sont mis en place qu'après le repliement des protéines. Or, pour la protéine prion, les choses semblent inversées, l'absence de pont disulfure empêchant le repliement correct de la protéine. Expérimentalement, si le pont disulfure n'est pas formé lors de la renaturation, la protéine précipite et forme essentiellement des feuillets $ß$. Toutefois, cette forme qui exhibe certaines caractéristiques structurales de la protéine pathologique n'est pas infectieuse, c'està-dire n'induit pas la maladie quand elle est injectée à un animal (voir glossaire). En outre, la protéine pathologique riche en feuillets $ß$ conserve son pont disulfure. Une autre condition expérimentale provoque l'apparition des feuillets $\beta$ et l'agrégation de la protéine : la renaturation dans des conditions acides (à $\mathrm{pH}$ inférieur à 4). Dans des conditions acides et partiellement dénaturantes (IM CIGdn) apparaît une structure riche en feuillets ß qui s'agrègent sous forme d'amyloïdes [7]. La cinétique d'agrégation est fortement dépendante de la concentration protéique, ce qui représente une propriété commune aux macromolécules qui sont peu solubles. Il faut noter que la formation d'amyloïdes résulte de la constitution d'interactions spécifiques entre des feuillets $ß$. La dépendance à la concentration pourrait être alors le résultat d'un phénomène de nucléation [8]. Une analyse fine de la dénaturation à $\mathrm{pH}$ acide de la forme repliée normalement à $\mathrm{pH}$ neutre a permis de révéler l'existence d'un intermédiaire de repliement qui est constitué de feuillets $ß$ et expliquerait la formation des agrégats amyloïdes. In vivo, on trouve généralement la forme agrégée dans les lysosomes qui sont des structures acides dont le $\mathrm{pH}$ peut être inférieur à 5. C'est pourquoi il a été envisagé que c'est lors du transport de la protéine vers ou dans le lysosome, pour être dégradée, que se produirait le changement de conformation pathologique.

Il apparaît donc que, dans des conditions acides et sous l'effet d'une dénaturation (plus ou moins partielle), la protéine prion passe d'une forme contenant essentiellement des hélices $\alpha$ en une forme qui contient principalement des feuillets $\beta$ et précipite sous forme amyloïdes. Cette structure présente en outre la propriété de résister à la protéinase $K$, tout comme la PrPsc, mais son infectiosité n'a pu être démontrée. Si le pH acide dans

Figure 3. Conversion de la PrPC en PrPsc in vitro dans un système acellulaire. A. Principe du test. La réaction utilise de la PrPSc naturellement résistante à la protéinase $K$, partiellement purifiée et extraite de cerveaux d'animaux infectés, et de la PrPC (PrPsen) marquée au ${ }^{35 S}$ radioactif et obtenue à partir du surnageant d'une culture de cellules transfectées avec une protéine ne pouvant fixer l'ancre GPI. Après incubation de plusieurs heures, le mélange réactionnel est soumis à une digestion par la protéinase $K$ puis à une électrophorèse, qui permet de visualiser la conversion de la $35 \mathrm{~S}$ PrPsen en $35 \mathrm{~S}$ PrPres. B. Résultats d'une analyse de conversion et de l'effet de barrière d'espèce. Le schéma représente un gel d'électrophorèse. Les protéines PrPsen de hamster $(H)$ ou de souris $(S)$ sont totalement dégradées par la protéinase K. En revanche, leur incubation avec de la PrPres provenant de la même espèce induit leur conversion en une forme résistante à la protéinase $K$, qui migre avec le marqueur de poids moléculaire de 20,9 kDa. On observe que si la PrPsen est incubée avec la forme résistante d'une espèce différente, sa conversion est fortement réduite : ce phénomène est appelé barrière d'espèce. En l'absence d'incubation avec la protéinase $K$, les deux formes PrPsen et PrPres migrent de façon semblable $(28,5 \mathrm{kDa})$. 
les lysosomes est bien la cause première qui induit le changement de conformation de la protéine, pourquoi ce phénomène est-il si rare ? Ainsi, cette explication, qui paraît logique au premier abord, ne fait donc que repousser le problème, et la question fondamentale de comprendre comment une même séquence peut donner deux structures reste entière. II faut supposer, soit que les deux structures correspondent à des environnements thermodynamiques différents, soit que le repliement de cette protéine comprenne une bifurcation et que la structure $\operatorname{PrPsc}$ soit très difficile à atteindre d'un point de vue cinétique. Les analyses de la stabilité relative des deux formes de la protéine, à $\mathrm{pH}$ acide pour la forme en feuillet $\beta$ et à $p H$ neutre pour la forme en hélice $\alpha$, favorisent la seconde hypothèse [9].

\section{Repliement in vivo : protéines chaperons}

Dans la cellule, le processus de repliement des protéines n'est certainement pas aussi simple qu'in vitro. La cellule qui ne peut se permettre un trop grand nombre d'erreurs a développé des systèmes de contrôle du repliement des protéines dont les protéines chaperons qui sont des molécules dont le rôle est d'aider au repliement des autres protéines [10]. La première étape est généralement une reconnaissance de la protéine pour ensuite l'amener à la bonne conformation. La participation de molécules chaperons au phénomène prion avait été postulée à partir de considérations théoriques [11]. Puis, il a été démontré que la protéine chaperon Hsp60 reconnaissait spécifiquement la protéine prion aussi bien dans un système double hybride de levure [12] que dans un système purifié [13]. En outre, l'interaction paraît si forte (l'énergie libre de l'interaction est supérieure à $22 \mathrm{~kJ} /$ mole, c'està-dire plus importante que l'énergie libre de stabilisation de $\operatorname{la} \operatorname{PrPc}$ ) que la dénaturation de la protéine prion a été suggérée [13]. Par ailleurs, la conversion in vitro, dans des systèmes non purifiés, voit son rendement augmenté de façon spectaculaire en présence de chaperons (voir ci-dessous). Enfin, des analyses de la barrière d'espèce en utilisant des protéines prion chimères ont montré que certains acides aminés devaient être impliqués dans une interaction avec une protéine inconnue $(\rightarrow) \quad$ nommée protéine $X(\rightarrow)$ par S.B. Prusiner [14]. Ce faisProtéine $X$ ceau de résultats suggère la participation d'une protéine Voir $\mathbf{m} / \mathbf{s} \quad$ chaperon lors du changement de conformation de la protéi2000, $n^{\circ} 10$, ne normale en protéine pathologique.

p. 1130 ne prion pathologique ( $\mathrm{PrPSc}$ ) a la capacité de convertir la protéine prion normale ( $\mathrm{PrPC}$ ) en protéine pathologique. La réalisation de cette conversion in vitro constitue donc une étape essentielle pour la validation de cette théorie. Conscient de ce fait, S.B. Prusiner a essayé dès 1992 d'obtenir cette conversion mais sans succès [15]. C'est au groupe de B. Caughey que I'on doit le premier succès... partiel [16]. La réaction utilise de la PrPsc partiellement purifiée, extraite de cerveaux d'animaux infectés, et de la $\operatorname{PrPc}$ (PrPsen) marquée au 355 radioactif et obtenue à partir du surnageant d'une culture de cellules transfectées avec une protéine ne pouvant fixer l'ancre GPI. Après incubation de plusieurs heures, la mesure de l'acquisition de la résistance à la protéinase $K$ dans la fraction radioactive est effectuée (Figure 3). On ne mesure donc pas réellement la conversion de la PrPC en PrPSc mais la conversion d'une forme sensible à la protéinase $K$ (PrPsen) en une forme résistante (PrPres). Une autre caractéristique de ce système est la nécessité d'utiliser un très large excès, d'environ 50 fois, de la forme PrPSc, ce qui rend très difficile la caractérisation de l'apparition de la propriété essentielle, l'infectiosité. Dans les premiers essais, le traitement de la PrPsc par un agent dénaturant, le CIGdn, est apparu essentiel. Avec l'amélioration du système, le recours à des sels plus physiologiques a permis des rendements corrects. Toutefois, les meilleurs résultats ont été obtenus en utilisant les protéines chaperons ; la protéine Hsp60 décuple le rendement de la réaction surtout si l'on évite la dénaturation par le CIGdn [17]. Un des grands succès de ce système de conversion a été la reproduction, dans des conditions acellulaires, du phénomène de souches et de la barrière d'espèce (Figure 3B). On sait en effet que les différentes souches peuvent être différenciées par électrophorèse après traitement par la protéinase $K$ mais aussi par leur plus ou moins grande facilité à reproduire la maladie. Le système de conversion in vitro reproduit avec suffisamment de fidélité les caractéristiques biochimiques (en particulier la mobilité électrophorétique) de souche apportées par la PrPsc [18]. Ce phénomène a été utilisé pour déterminer si la PrPres obtenue était infectieuse, mais le résultat s'est avéré négatif [19], la protéine transconformée obtenue in vitro n'est pas infectieuse.

Tout récemment un nouveau système de conversion in vitro a été décrit [20]. II s'agit d'une amplification cyclique dont un certain nombre de caractéristiques méritent d'être analysées. La figure 4 schématise le principe du système. II est fondé sur deux idées nouvelles. La première consiste à " casser " les agrégats amyloïdes ( $\mathrm{PrPsc}$ ) en petits fragments qui seront autant de noyaux de "cristallisation". La seconde consiste à 
ajouter un lysat de cerveau d'animal sain comme source de protéine normale $\operatorname{Pr} P C$. A chaque cycle, la quantité de protéine résistante à la protéinase $K$ (PrPres) augmente. On obtient ainsi une amplification exponentielle, proche dans son principe de l'amplification de l'ADN par PCR ; c'est la raison pour laquelle les auteurs ont donné à cette méthode le nom de PMCA (protein misfolding cyclic amplification) que l'on peut traduire littéralement par "amplification cyclique du mal-repliement protéique". La conséquence immédiate de ce système est de pouvoir multiplier la quantité de protéines résistantes à la protéinase $K$ et donc d'augmenter considérablement la sensibilité des tests de détection. Contrairement au système de B. Caughey, qui utilisait des protéines partiellement purifiées, celui-ci utilise des homogénats de cerveau et a permis de révéler que la présence de ce lysat est nécessaire à la formation de PrPres. Cette observation suggère l'existence d'un co-facteur, impliqué dans la conversion de la protéine normale ( $P r P C)$ en protéine résistante à la digestion par les protéases (PrPres). S'agit-il d'une protéine chaperon comme nous l'avons discuté plus haut? Toutefois, la question principale posée par le développement de ce système est bien évi-

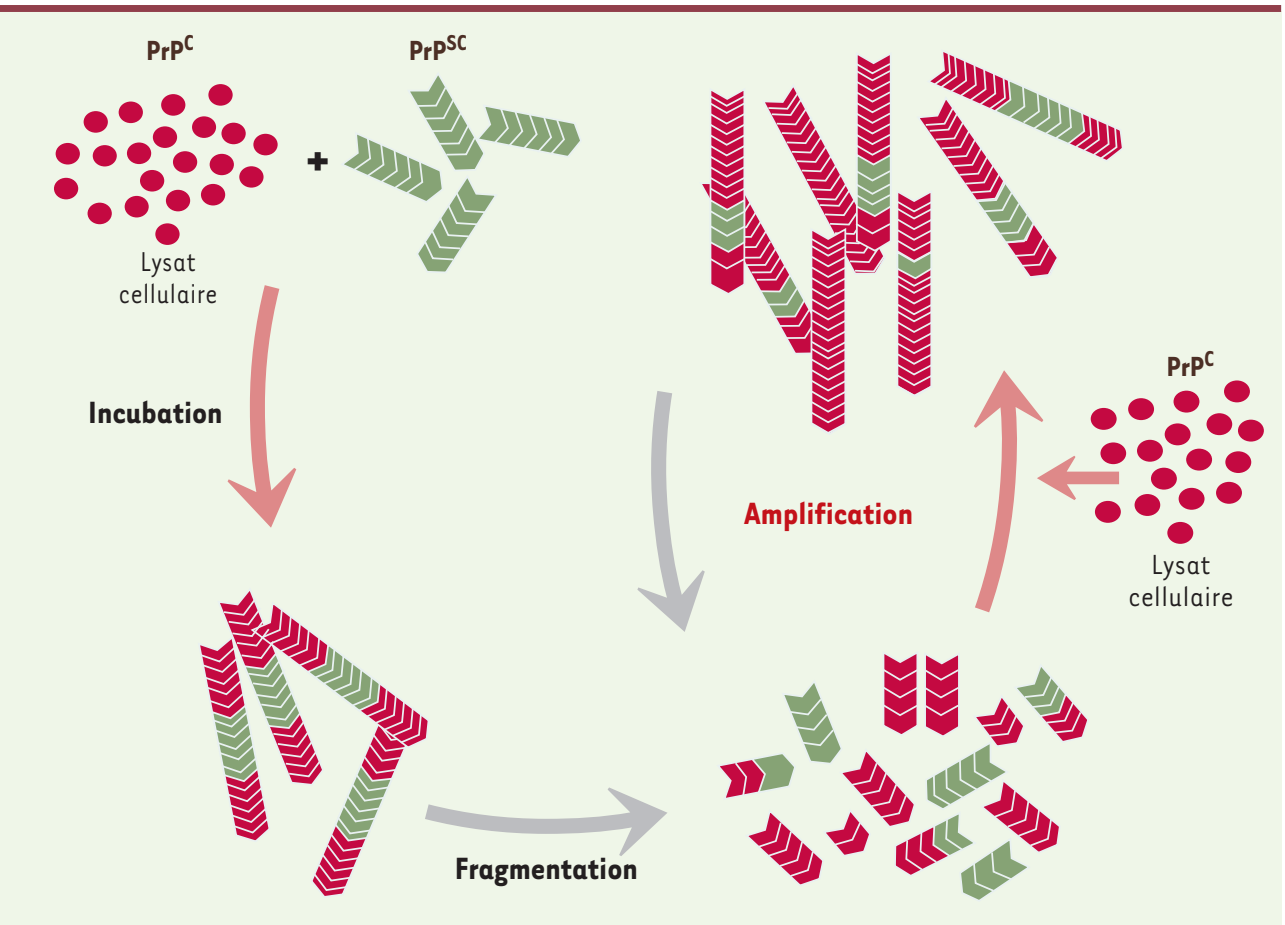

Figure 4. Schéma représentatif du système de Claudio Soto (protein-misfolding-cyclic-amplification, PMCA). Un excès de protéine PrPC est incubé en présence de PrPSc. Les agrégats amyloïdes sont ensuite fragmentés par sonication, puis incubés à nouveau avec de la PrPC. A chaque cycle d'incubation et de fragmentation, la quantité de protéine résistante à la protéinase $K$ augmente en raison de l'incorporation de monomères de PrPc dans l'amyloïde. Cette expérience conduit à une amplification de type exponentiel de la forme amyloïde résistante à la protéinase $K$. demment celle de la nature infectieuse de la protéine formée, question dont la réponse est attendue avec impatience.

\section{Pourquoi est-il (encore) impossible de rendre infectieuse une protéine prion recombinante?}

Conceptuellement, la démonstration de la conjecture prion, c'est-à-dire l'existence d'une protéine infectieuse, paraît simple. Il suffirait de prendre une protéine recombinante purifiée et de la rendre infectieuse. Cette expérience n'a jamais été couronnée de succès. Si l'on accepte l'hypothèse prion, plusieurs explications à ces échecs peuvent être envisagées. La première suppose que la structure spatiale de la forme infectieuse est très difficile à atteindre et que les conditions physico-chimiques spécifiques permettant d'y parvenir n'ont pas encore été trouvées. De nombreux laboratoires utilisent cette hypothèse de travail mais, pour l'instant, sans succès. Une seconde possibilité est l'existence d'un "co-facteur" qui n'a pas encore été identifié. Des protéines chaperons aux acides nucléiques spécifiques, les candidats sont nombreux mais, ici encore, aucun n'a pu résoudre le problème.

Ainsi, en dépit des nombreux progrès réalisés ces dernières années sur

la biologie de la protéine prion, la démonstration formelle de l'hypothèse prion constitue encore un défi dont l'importance n'a pas besoin d'être soulignée. $\diamond$ 


\section{SUMMARY}

The prion protein : structure, dynamics

\section{and in vitro conversion}

The prion hypothesis of S.B. Prusiner has challenged not only the molecular biology but also the main paradigms of structural biology. Indeed, the prion hypothesis supposes that : 1) a protein can adopt two different conformations and 2) that the rogue isoform can convert the normal isoform to its own structure. We review the present knowledge on the spatial structure and folding characteristics of the prion protein. Atomic structure of the recombinant prion protein has been solved by NMR. It is constituted of a core mainly under $\alpha$-helix conformation and a long unstructured $\mathrm{N}$-terminal. This spatial structure does not reveal specific any features that would simply explain the existence of the two structures. The folding studies show a very simple pathway when operated under neutral $\mathrm{pH}$, however at acidic $\mathrm{pH}$ the protein can adopt a ß-sheeted secondary structure that results in amyloïd formation, unfortunately this form is not infectious. It is known that in vivo, chaperones assist protein folding. Many experimental results suggest the participation of chaperones to the prion genesis, but we still can neither produce infectious protein nor understand how these chaperones could participate to the phenomenon. In vitro conversion of the normal is oforme to a proteinase $\mathrm{K}$ resistant protein has been successfully designed, but has not yet produced an infectious protein. Altogether, the experimental results do not discount the structural aspects of the prion hypothesis but have failed to confirm it. Some new working hypotheses are being still examined by researchers. $\Delta$

GLOSSAIRE operationnelle.

La notion de souche pour l'agent infectieux "prion" ressemble fortement à celle définie pour les virus. II s'agit d'isolats qui transmettent à leur descendance des caractéristiques qui, dans ce cas, sont le temps d'incubation et la répartition des lésions dans le cerveau. Les propriétés des souches se transmettent même après passage d'une espèce à l'autre. Par exemple, la souche de l'£SB (encéphalopathie spongiforme bovine) est la même que celle trouvée chez les personnes atteintes de nvMCJ (nouveau variant de la maladie de Creutzfeldt-Jakob), et présente les même caractéristiques une fois transmise à des souris syngéniques. Dans le cadre de l'hypothèse prion (protéine seule), les caractéristiques de souche sont cryptées dans la structure spatiale de la PrPsc (voir texte).

Protéine infectieuse La démonstration de l'hypothèse prion serait la mise en évidence de l'infectiosité de la protéine prion purifiée produite dans des conditions axéniques (par exemple, la protéine recombinante). Expérimentalement, cela signifie que son injection à un animal devrait produire la maladie et celle-ci devrait être transmissible à d'autres animaux. 
Amyloïdes Certaines protéines forment des complexes qui résultent de l'association intermoléculaire de feuillets $B$. Ces structures nommées amyloïdes présentent une organisation bidimensionnelle que l'on peut caractériser par des réactions chimiques spécifiques. La biréfringence du Rouge Congo, qui s'insère dans la structure amyloïde, est très caractéristique des amyloïdes.

\section{Formes génétiques humaines des maladies à prion}

Chez l'homme, on distingue trois étiologies dans les maladies à prion : les formes infectieuses, les formes sporadiques et les formes génétiques. Plusieurs syndromes sont associés aux formes génétiques (Creutzfeldt-Jacob, Gersmann-Straussler-Scheinker et insomnie fatale familiale) mais ils sont tous associés à des mutations dans la protéine prion.

La pénétrance est très forte, voire complète.

\section{RéFÉRENCES}

1. Prusiner SB. Prions. Proc Natl Acad Sci USA 1998; 95 : 13363-83.

2. Laurent M. Les maladies à prion : I'hypothèse de la "protéine seule" et ses conséquences dynamiques. Med Sci $1996 ; 12: 774-85$.

3. Caughey B., Interactions between prion protein isoforms: the kiss of death? Trends Biochem Sci $2001 ; 26$ : 23542.

4. Riek R, Hornemann S, Wider $G$, Billeter M, Glockshuber R, Wüthrich K. NMR structure of the mouse prion protein domain PrP (121-231). Nature 1996 ; 382 : 180-2.

5. Liemann S, Glockshuber R. Influence of amino acid substitution related to inherited human prion diseases on the thermodynamic stability of the cellular prion protein. Biochemistry 1999 ; 38 : 3258-67.

6. Wildegger G, Liemann S, Glockshuber R. Extremely rapid folding of the $\mathrm{C}$-terminal domain of the prion protein without kinetic intermediates. Nat Struct Biol 1999; $6: 550-3$.

7. Swietnicki W, Morillas M, Chen $S$, Gambetti P, Surewicz WK. Aggregation and fibrilization of the recombinant human prion protein huPrP90-231. Biochemistry 2000 ; 39 : 421 31.

8. Come JH, Fraser PE, Lansbury
PT. A kinetic model for amyloid formation in the prion diseases: importance of seeding. Proc Natl Acad Sci USA $1993 ; 90$ : 5959-63.

9. Baskakov IV, Legname G, Prusiner SB, Cohen FE. Folding of prion protein to its native a-helical conformation is under kinetic control. J Biol Chem 2001 ; 276 : 19687-90.

10. Morange M. Protéines chaperons. Med Sci $2000 ; 16$ : 630-4.

11. Liautard JP. Prions and molecular chaperones. Arch Virol $1993 ; 7$ : 227-43.

12. Edenhofer $F$, Rieger $R$, Famulok M, Wendler W, Weiss $S$, Winnacker $\varepsilon$ L. Prion protein PrPC interacts with molecular chaperones of the Hsp60 family. J Virol 1996; $70: 4724-8$.

13. Guerin MC, Bettache $S$, Aumelas A, Chiche L, Liautard JP. Use of surface plasmon resonance to analyse recombinant prion protein interaction with molecular chaperones. Int J Biochromatogr 2001

14. Telling GC, Scott $M$, Mastrianni J, et al. Prion propagation in mice expressing human and chimeric PrP transgenes implicates the interaction of cellular PrP with another protein. Cell $1995 ; 83: 79-90$.

15. Raeber AJ, Borchelt DR, Scott M, Prusiner SB.
Attempts to convert the cellular prion protein into the scrapie isoform in cell-free systems. J Virol $1992 ; 66$ : 6155-63.

16. Caughey BW, Kocisko DA, Raymond GJ, Lansburry PT. Aggregates of scrapie-associated prion protein induce the cell-free conversion of protease-sensitive prion protein to the proteaseresistant state. Curr Biol $1996 ; 2: 807-17$.

17. Deburman SK, Raymond GJ, Caughey B, Lindquist $S$. Chaperone-supervised conversion of prion protein to its protease-resistant form. Proc Natl Acad Sci USA 1997 ; 94 : 13938-43.

18. Horiuchi M, Priola SA, Chabry J, Caughey B. Interactions between heterologous forms of prion protein : binding, inhibition of conversion and species barriers. Proc Natl Acad Sci USA 2000 ; 97 : 5836-41.

19. Hill AF, Antoniou M, Collinge J. Protease-resistant prion protein produced in vitro lacks detectabble infectivivty. J Gen Virol 1999 ; 80 : 11-4.

20. Saborio GP, Permanne B, Soto C. Sensitive detection of pathological prion protein by cyclic amplification of protein misfolding. Nature $2001 ; 411: 810-3$.

\section{TIRÉS À PART \\ J. P. Liautard}

